\title{
Antigen Presentation Interaction
}

National Cancer Institute

\section{Source}

National Cancer Institute. Antigen Presentation Interaction. NCI Thesaurus. Code C40475.

Antigen Presentation Interaction involves specific non-covalent interaction (binding) through intermolecular physical forces of attraction and spatial complementarity of $\mathrm{T}$ Cell Antigen Receptors with proteolytically processed fragments of antigenic foreign proteins displayed in association with self $\mathrm{MHC}$ antigens on the surface of antigen presenting macrophages. 